\title{
Misuses of Clustering and Absolute Scales of Measurement in the Analytic Hierarchy Process
}

\author{
Kirti Peniwati \\ Institute for Management Education and Development \\ Jl. Menteng Raya 9, Jakarta 10340, Indonesia
}

\begin{abstract}
The grouping of elements, called clustering, is a fundamental operation in the Analytic Hierarchy Process. What elements are chosen and how they are clustered and located in a hierarchy have a profound effect on the outcome and the conclusions derived from the outcome. A valid AHP hierarchy must represent a problem well, and also ensure that all AHP axioms are satisfied. Examples are given to show how violations of the AHP axioms can lead to wrong priorities, infeasible interpretations, and erroneous conclusions. I also show that a mechanical approach to priority setting can mislead one to believe that the theory needs to be modified to accommodate one's commitment to an arithmetic procedure rather than the other way around.
\end{abstract}

Keywords: Analytic Hierarchy Process, clustering, absolute measurement, relative measurement, multicriteria decision making

\section{Introduction}

The Analytic Hierarchy Process (AHP) seeks to abstract the essential flow of influences in a problem with a hierarchic structure to determine the most likely or desired outcome. The dominance of influence is elicited through pairwise comparisons of relative importance. From the comparisons, a ratio scale of relative importance or priorities is derived. Synthesis involves the weighting and addition of the weighted values of an element by the weights of its parent properties.

Clustering in the AHP is the process of classifying and grouping the identified influences such that elements in a cluster serve a common goal, have a property(ies) in common, or collectively contribute to the function of a more general element(s). It is also the process of dividing a cluster, containing a large number of inhomogeneous alternatives, into small homogeneous (sub)clusters. The former is used to represent a problem as a hierarchy, the latter is used to create a scale across inhomogeneous elements and to improve the efficiency and consistency of the judgments.

Structuring a hierarchy involves identifying the influences that affect the outcome of a problem, clustering them, ordering the ciusters into descending levels of decreasing importance, and arranging them in different levels of the hierarchy according to their preordered relative importance and their influence relationships. Briefly then, an AHP hierarchy is a structural arrangement of the elements of a problem in clusters, and of clusters in different levels. Higher level elements transmit influence to lower level ones, or, lower level elements contribute to the functioning of the higher level ones.

Following the systems approach, the function of a higher level element is not simply the 
sum of those contributed by the elements below it in the hierarchy. It can be more because of the synergistic functioning of the lower level elements, or it can be less because the elements can diminish the influence of other elements. The idea that the whole should be equal to the sum of its parts is an untenable idea in hierarchic thinking. In organizational theory, for example, group learning is not the sum of the learning of its members, nor is the capability of an organization the sum of all individual skills and resources the organization has. Today there are cosmologists dealing in the theory of "cold-dark-matter" who maintain the amazing idea that the weight of the universe as a whole is more than the weight of its components. This is attributed to undetectable particles that amplify the effect of the original density fluctuations that form the universe [11].

Proper hierarchical structuring in the AHP assures fulfillment of the AHP axioms. Following manipulations that violates the axioms implies that the manipulations are unsuitable for the AHP. A different theory may justify the manipulations but it would have its own axioms and is no longer the AHP. The AHP axioms that are directly relevant to hierarchical structuring of a problem are:

(1) Valid representation of a problem from the point of view of the decision maker (expectations). A given problem may still be interpreted differently by different people, gives different outcomes and interpretations.

(2) Proper clustering of elements and arranging of clusters to represent outerdependence of lower level elements on higher level ones.

(3) Careful clustering of homogeneous elements to ensure greater consistency in the comparisons of the elements.

Appropriate questions 'must be asked to elicit the right kind of judgments (axioms of independence and of expectations). Our contention here is that misunderstanding of the AHP has often arisen out of what I call misclustering which I will now examine in detail.

\section{Clustering in the AHP}

The purpose of the entire process of dealing with a hierarchic process is to derive the rank order of all the elements and in particular the alternatives at the bottom level of the hierarchy. A step that precedes numerical ordering (by pairwise comparisons) is the structural ordering of the elements and their clusters based on experience and judgment. In this process, the elements are arranged in a level of a hierarchy according to generality and precedence in influence.

Numerical ordering is less subjective than structural ordering in the sense of converting judgments from pairwise comparisons to numerical values and proceeding formally to derive priorities. Structural ordering is made more objective in the basic theory of the AHP through suggestions made from a large class of hierarchies examined as to what should go at what level to best capture its influence in the outcome. The process of prioritization will generally involve identification and grouping of elements, followed by a further decomposition of the groupings until the appropriate level of detail is adequately captured by the structure to yield the desired 
refinement or accuracy in the resulting numerical priorities.

Throughout the process, one incurs the risk of grouping together elements that are dissimilar according to some property or if they are similar, they may not be of comparable magnitudes. In the end, one will discover the similarities by making the comparisons. The crucial step in deciding the accuracy of the process is the comparison of elements or group of elements in one level, with respect to some or all the elements in the level immediately above used as attributes or properties of these elements. (The word attribute here is generic, and could mean a property, a cause, an effect, or an influence of a certain kind).

The AHP requires that a large number of inhomogeneous alternatives be compared by grouping them into small and homogeneous subclusters. Unlike placing elements in clusters, here we assume no synergistic function of the subclusters, and the operation does not add new elements or new levels in the hierarchy. A subcluster arbitrarily chosen need not serve as a meaningful criterion in the hierarchy. To simply deal with an aggregate of words indicating different things does not automatically grant meaning to the collection. The AHP adopts words to represent criteria that give meaning to the flow of influence. For example, bundles of words in a subcluster like the names of cities with the same direction from Singapore does not convey how the direction influences the distance of the cities from Singapore [12]. That is why I believe, and will show further below, that some authors have been confused about how the process of prioritization works in the AHP.

\section{Different Perceptions About a Given Problem - An Example}

The first step in approaching a problem with the AHP before identifying a hierarchy is to determine the goal(s) or supercluster(s) and there can be a difference in opinion about them leading to different outcome. For example, the benefits of an undertaking, its costs, or its risks all have different goals that would generally require a separate hierarchy. The outputs of these hierarchies would then be appropriately combined to obtain the final outcome $[4,6,7]$.

An example for applying the AHP to benefit-cost analysis in a decision to cross a river is proposed by Saaty [4, pp. 115-120; 8, pp. 183-186]. It includes income in the benefits hierarchy and capital and operating and maintenance in the costs hierarchy, together with other intangibles. Depending on what goal is served, decision makers may have different opinions about what dollar measurements should be included and what hierarchical structure is most appropriate for the problem. There are three possible perceptions as what goal to pursue. Each will have a different outcome with respect to the financial criteria:

(1) To rank new projects according to their potential ability to 'multiply' each dollar spent on them. In this case, income will be included in the benefits hierarchy and costs in the costs hierarchy. The highest ranked project will be the one with the highest income to cost ratio.

(2) To rank new projects according to their potential to generate net cash inflows in absolute terms. In this case, only one element of net cash flow (obtained by subtracting cost from income) is required, and located in the benefits hierarchy. Obviously, only projects with positive cash flows will be considered. The highest ranked project will be the one with 
(3) To rank expansions of existing projects according to their ability to generate income for each additional dollar invested in the projects. In this case, marginal analysis is needed $[7$, p. 153]. The highest ranked project will be the one with the highest incremental income to cost ratio.

The three analyses are all valid AHP models. However, they will generally give different results, and the priority values derived will have different meanings. In the case of group decision making, the AHP methodology facilitates the process of aligning group perceptions about the problem and the specific goal to be achieved. Different understandings would lead to disagreements on the structure of the hierarchy or on the pairwise comparison judgments. The entire process of decision making using the AHP is a communication process to align perceptions among those involved in the process.

According to Saaty [7, pp. 153-155], to conduct marginal benefit-cost analysis with the AHP, one must arrange the projects in such a way that their synthesized cost priorities are in increasing order, and form the ratios of the differences between benefits priorities and those of cost priorities $\left(b_{i+1}-b_{i}\right) /\left(c_{i+1}-c_{i}\right)$. Projects with negative ratios, i.e. projects that give less additional benefits than those given by the projects that require less further investments, are deleted as potential options.

Bernhard and Canada [1] have examined whether the proposed benefit-cost approach works for a simple problem where only dollar values are considered. They take the rivercrossing example and give the dollar income and cost data to match the priorities in the example. They say: "The best alternative is then, clearly, the one which offers the highest net difference of benefits minus costs ... Presented below is an incremental benefit/cost ratio ritual which is guaranteed to make that correct choice". They did not realize that the analysis they intended to do and the one they actually did were not the same. They intended to select a project which offered the highest net difference of income and cost. They did incremental analysis but compared the outcome with that of the benefit to cost analysis done by Saaty. They do not get the same results, and incorrectly conclude that there are some problems in using the benefit to cost ratios with the AHP.

The same example is used here to show how three different perceptions as to what one is looking for lead to different outcomes and interpretations. Following those authors' approach, the income and cost data are used directly to give accurate and consistent judgments. The derived priorities equal the normalized data (uses only dollars). Conversion from absolute to relative measurements before and after manipulation can lead to different resuits. This is not a problem of the AHP, but of a misuse of the arithmetic of measurement on a single criterion and its constituents. Such measurement on the same scale must be all combined into a single answer before combining with other scales [7].

1. The first perception: selecting a project with the highest unit benefit to unit cost.

The pairwise comparison judgments are in response to the question: With respect to the goal, which project benefits more (or costs more), and how much more? 


\begin{tabular}{|c|c|c|c|c|c|}
\hline \multirow{2}{*}{ Project } & \multicolumn{2}{|c|}{ Benefit } & \multicolumn{2}{|c|}{ Cost } & \multirow{2}{*}{$\begin{array}{c}\text { Benefit } \\
\text { to Cost } \\
\text { Ratio }\end{array}$} \\
\hline & $\$ M$ & $\begin{array}{l}\text { Relative } \\
\text { Weight }\end{array}$ & $\$ M$ & $\begin{array}{l}\text { Relative } \\
\text { Weight }\end{array}$ & \\
\hline 1. The Ferry & 10 & 0.067 & 5 & 0.050 & 1.34 \\
\hline 2. The Bridge & 50 & 0.333 & 30 & 0.300 & 1.11 \\
\hline 3. The Tunnel & 90 & 0.600 & 65 & 0.650 & 0.92 \\
\hline
\end{tabular}

This analysis indicates that the ferry project is the best alternative. It means that investment in the ferry is expected to yield the highest return among projects. For each dollar invested, the ferry project is expected to generate income 1.34/1.11 and 1.34/0.92 times more than that generated by the bridge and by the tunnel projects respectively.

2. The second perception: selecting a project with the highest net cash inflow (benefits minus costs).

The pairwise comparison judgments are in response to the question: With respect to the goal, which project gives more absolute net cash flow, and how much more?

\begin{tabular}{|l|c|c|c|c||}
\hline Project & Benefit & Cost & $\begin{array}{c}\text { Benefit } \\
\text { minus } \\
\text { Cost } \\
\$ \mathrm{M}\end{array}$ & Weight \\
\hline 1. The Ferry & 10 & 5 & 5 & 0.10 \\
2. The Bridge & 50 & 30 & 20 & 0.40 \\
3. The Tunnel & 90 & 65 & 25 & 0.50 \\
\hline
\end{tabular}

This analysis gives the tunnel project as the most preferred alternative. It implies that the tunnel would generate the highest absolute net income among the alternatives considered. The net income would be $0.5 / 0.1$ and $0.5 / 0.4$ times more than that generated by the ferry and by the bridge projects respectively.

3. The third perception: allocating additional investment to existing projects.

The judgments are in response to the question: with respect to the goal, which project gives greater additional income (or requires additional investment), and how much greater? Here one must be careful in adding normalized priority weights [7, pp 310-320; 9]. The marginal ratios calculated using absolute data is different from the ratios calculated using relative measures because of the different normalization constants between income and costs. Although multicriteria benefit/cost analysis uses derived priorities to calculate the marginal benefit to cost ratio, the single criterion problem in this example must be solved using the absolute data. They 
are different from that given by Bernhard and Canada [1], who calculate them using the priority values.

\begin{tabular}{||c|c|c|c|c|c|}
\hline \multirow{2}{*}{ Project } & \multicolumn{2}{|c|}{ Incr. Benefit } & \multicolumn{2}{c|}{ Incr. Cost } & $\begin{array}{c}\text { Incr. } \\
\text { Benefit } \\
\text { to Cost } \\
\text { Ratio }\end{array}$ \\
\cline { 2 - 6 } & $\$ \mathrm{M}$ & Weight & $\$ \mathrm{M}$ & Weight & \\
\hline 1. The Ferry & 10 & 0.111 & 5 & 0.077 & 1.44 \\
2. The Bridge & 40 & 0.444 & 25 & 0.385 & 1.15 \\
3. The Tunnel & 40 & 0.444 & 35 & 0.539 & 0.82 \\
\hline
\end{tabular}

This analysis gives the same ranking as that based on the unit benefit to unit cost ratio, but the priority values are different and must be interpreted differently. It implies that with the same amount of additional investment in the existing projects, the ferry is expected to give an added income of $1.44 / 1.15$ and $1.44 / 0.82$ times more than that of the bridge and the tunnel respectively.

Their derived priorities give the same rank but different weights as seen below:

\begin{tabular}{||l|c|c|c|}
\hline Project & $\begin{array}{c}\text { Incr. Benefit } \\
\text { Weight }\end{array}$ & $\begin{array}{c}\text { Incr. Cost } \\
\text { Weight }\end{array}$ & $\begin{array}{c}\text { Incr. Benefit } \\
\text { to Cost Ratio }\end{array}$ \\
\hline 1. The Ferry & 0.067 & 0.050 & 1.34 \\
2. The Bridge & 0.266 & 0.250 & 1.06 \\
3. The Tunnel & 0.267 & 0.350 & 0.76 \\
\hline
\end{tabular}

\section{Multicriteria Problems with the Same Underlying Measurement - An Example}

Multicriteria problems are single criteria (transformable) problems in the AHP if they have the following fundamental characteristics [3]:

(a) The criteria share the same (or can be mapped with a scaling factor to the same) underlying unit.

(b) The perception of the decision maker of the common unit is constant across all mapped criteria.

(c) The alternatives are homogeneous with respect to each criterion.

(d) The alternatives can be individually measured with some absolute scale or intensity rating with respect to each criterion.

In a given problem, all tangible criteria meeting the above characteristics must be combined first, before comparing them with other tangibies with different measurements and with intangibles. Saaty [7, pp 306-308] shows an example of a family choosing the best house, having four factors dominating their thinking: the price (\$), the remodelling costs $(\$)$, the size 
(sq.ft), and the style (intangible). The first two factors have the same measurements, so they must be combined and converted into a ratio scale. Although four factors are considered, it is a three criteria AHP problem of choosing the best house according to cost, size, and style.

Schoner and Wedley [10] give an example of buying a car with the objective of paying the minimum cost over the life of that car (5 years). The car characterics affecting the total cost are purchase price, yearly maintenance cost, and fuel usage. Although the criteria have different measurements, they are all mapped by the authors into dollars, the unit of the objective. The measurements of the cars in each factor are:

\begin{tabular}{|c|c|c|c|}
\hline \hline Car & $\begin{array}{c}\text { Price } \\
\$\end{array}$ & $\begin{array}{c}\text { Maintenance } \\
\text { \$/ year }\end{array}$ & $\begin{array}{c}\text { Fuel } \\
\text { gal. / mile }\end{array}$ \\
\hline 1 & 14,000 & 2,000 & 0.05 \\
2 & 5,000 & 4,000 & 0.03 \\
3 & 6,000 & 4,000 & 0.05 \\
\hline
\end{tabular}

Scale factors are used to convert criteria measurements into dollars, using the following information: the car is to be driven 10,000 miles a year, and the cost of gasoline is estimated to be $\$ 1.50$ a gallon. The scale factor for price is 1 (total initial cost $=p \$$ ), for maintenance is 5 (total maintenance cost $=m \$ /$ year $\times 5$ years $=5 \times \mathrm{m} \$$ ), and for fuel consumption is 75,000 (total fuel cost $=f$ gallons/mile $\times 10,000$ miles/year $\times 5$ years $\times 1.5$ $\$$ /gallon $=75,000 \mathrm{x} \mathrm{f} \$$ ). The correct answer to the problem was found by directly calculating the total cost for each car and was normalizing the results to get relative numbers.

The authors then again solved the problem using the AHP approach. They showed that to get the correct answer, "the relative importance of a criterion must be proportional to the product of its scaling factor and the sum (or average) of the absolute values of option measurements on that criterion" [10].

$$
\mathrm{x}_{\mathrm{k}}=\mathrm{K} \cdot \mathrm{q}_{\mathrm{k}}: \sum_{\mathrm{k}} \mathrm{T}_{\mathrm{h}, \mathrm{k}}
$$

$x_{k}$ is the weight of the $k^{\text {th }}$ criterion, $K$ is the constant of proportionality, $q_{k}$ is the scaling factor of the $k^{\text {th }}$ criterion, and $\Sigma_{h} T_{h, k}$ is the sum of the absolute measurements of the alternatives under the $\mathrm{k}^{\text {th }}$ criterion. They found that the criteria weights are dependent on the measurement of the alternatives, and concluded that the AHP axiom that criteria are not outer dependent of the alternatives is violated. They supported their argument by showing that adding a copy of an existing alternative causes rank reversal if the weight of the criteria are maintained following the AHP axiom.

This car problem is an AHP single criterion problem [2,3,7]. Asking a meaningful question of the reiative importance of the criteria with respect to the goal is useful to indicate for the decision maker that this is not an AHP multicriteria problem. If the decision maker was asked: "With respect to selecting the car to purchase, which criterion is more important, and how much more?", he probably would say: "I don't care, as long as I get the car with the lowest total cost". His only concern is with the total cost, not how the cost is distributed to the different cost elements. 
It would become a multicriteria problem, however, if the decision maker does not perceive that the value of one dollar is the same for all the criteria. It is conceivable, for example, that one would be willing to pay more for purchase price than for maintenance or fuel because more operational costs implies more hassle (sending the car for maintenance and buying gas more often). If this is the case, and the decision maker still wants to use the objective data available, he may want to represent the problem into, say, a two criteria hierarchy of selecting the best car according to cost and inconvenience, and to break the inconvenience criterion down into maintenance inconvenience and fuel consumption. People need to understand this idea well to move on from the hangup about using numbers and converting absolute scales to relative values.

\section{Clustering Inhomogeneous or a Large Number of Elements}

If a cluster of elements to be pairwise compared does not fall within one order of magnitude, or if the number of elements is large, they must be compared in subclusters of a small number of homogeneous elements connected by a pivot or linchpin element [7].

(1) Divide the cluster into a number of subclusters of increasing size elements with a small number of elements in each subcluster (to do this, make a first pass or use AHP absolute measurement to order the elements gradually to improve the sorting).

(2) Pairwise compare and derive priorities for the elements in the first (smallest elements) subcluster.

(3) Take the highest priority element and inciude it in the second subcluster.

(4) Pairwise compare the elements in the second subcluster and derive their priorities.

(5) Divide the priorities of the elements in the second subciuster by the priority of the pivot in that subcluster and multiply them by the priority of the pivot in the first cluster. Now the pivot has equal priority in both clusters. It can be discarded from the second subcluster. The two subclusters can now be merged.

(6) Repeat the process in succeeding subclusters until the priorities of the elements in all subclusters are obtained.

(7) Renormalize the weight of all the elements.

The rule to follow in clustering homogeneous elements is to put them in small groups and compare them using any element as a pivot.

Sometimes one may want to (sub)cluster alternatives according to some attribute to facilitate thinking, and to obtain the relative weight of the (sub)clusters to be used to derive the overall weight of the alternatives instead of using the clustering procedure described above. Unfortunately, doing so may lead to a serious misapplication of the AHP. Here is an example.

Wedley, Schoner, and Choo [12] apply the AHP to estimate the relative distance of several cities from Singapore. They subcluster the cities according to their general direction from Singapore (northeast, northwest, and southeast), and employ two different methods. First, by treating the general direction from Singapore as a criterion and second, by treating the subclusters simply as a group of cities and then applying the AHP clustering procedure described 
above. They then compare the effect of adding or deleting cities, and find that rank reversal occurs with the first method, but not with the second!!

The diagram below shows their numerical results for the first method, the one that treats subciusters as criteria and shows rank reversal. The weights of the cities in the first rows are the initial local and global priorities that match the actual distance of the cities from Singapore. The weights in the second rows are the outcome after deleting three cities that give incorrect global priorities.

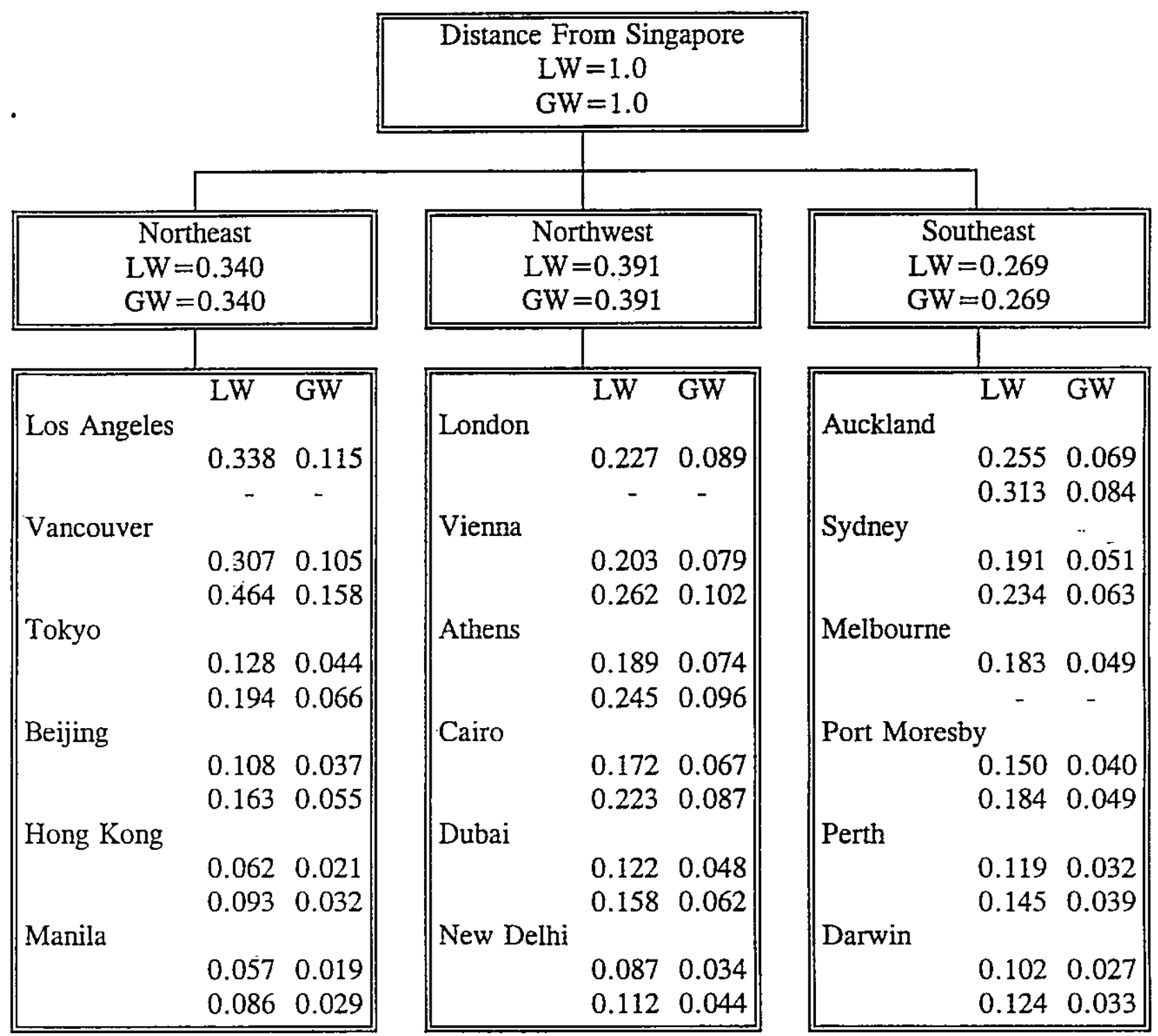

The authors analysis proceeds as follows:

(1) They pairwise compared the cities in each subcluster, by computing the ratios of their actual distance from Singapore. Here the judgments were accurate and consistent, assuming that they were the response to the question: which city is farther from 
Singapore and how much farther? Note that the question was formulated without referring to the general direction of the cities from Singapore,

(2) They used the mean (or the total) of the actual subcluster distances to compute the weight of each subcluster, assuming that they were the priorities that would have been derived in response to the question: which subcluster is farther from Singapore, and how much farther? The authors pointed out that the answer to this question was dependent on the alternatives, thus violating the AHP axiom of independence of higher level elements from the lower level ones,

(3) They studied the effect of adding or deleting alternatives, by maintaining the weight of the 'criteria' (claiming that it was following the AHP axiom).

They observed rank reversal and proposed that the AHP axiom, that higher level elements be independent of the lower level ones, be relaxed to prevent rank reversal. This is a serious misapplication of the AHP. It uses priorities derived from the pairwise comparison of a set of elements to mechanically determine the importance of a higher level criterion. In the AHP, the importance of a criterion in a hierarchy is determined by its relative importance among all the criteria, with respect to the higher goal, not from its descendants. This is not to say that alternatives cannot influence the importance of the criteria in all cases, because they can, but it must be dictated by the problem itself. If such feedback exists, then one should use the network approach of the AHP and deal with this kind of dependence with the supermatrix. One can use the hierarchic composition method described by Wedley et.al., as long as one realizes that he or she is dealing with a "dummy criterion" that has nothing to do with any of the AHP axioms. An interesting question to address would be how a decision maker would respond to the authors' question: "With respect to the goal, which subcluster is farther from Singapore, and how much farther?". Would he do some averaging to answer it or would he have some knowledgeable judgment about it? Whether or not people in general can respond to questions like this would determine the feasibility of the method for pairwise comparing clusters using the hierarchical composition approach proposed by the authors.

As Schenkerman [9] observes: "Experience reveals that some - if not many - decision makers, and at least some decision scientists, use weight incorrectly and inappropriately ... In consequence they make the wrong decisions". The $[0,1]$ scales derived from pairwise comparing clusters of elements are not the same. The $[0,1]$ interval is equivalent to the $[0,100]$ interval, of which one must ask: percent of what? In this example, the weight of a city shows percent of the distance of the city from Singapore to the total distance of all the cities. Adding or deleting cities from the cluster changes the total distance, deriving a different $[0,1]$ scale. It does not make sense to assume that numbers derived from pairwise comparing a cluster of elements can be used as priorities in a hierarchy that involves a different cluster.

The AHP requires one to ask meaningful questions to elicit pairwise comparison judgments. For example, with respect to a criterion directly above, one asks: which element is more important, and how much more? In the foregoing example, it is meaningless to ask: with respect to the northeast direction from Singapore, which city is more preferable, and how much more? The authors avoid answering the question by referring it directly to the goal instead. In his guidance on how to strùcture a hierarchy, Saaty [5] writes that inability to ask such a meaningful question means that more level(s) need to be inserted in the hierarchy. Using 
the same logic, elements in a level of the hierarchy are not meaningful elements as intended by the AHP if such questions cannot be formulated and answered.

\section{Adding Contingency Elements in a Hierarchy}

One can improve the validity of the hierarchic representation of a problem by including an element called the "unknown" at different levels of a hierarchy and a sensitivity test made of how much influence it would have on the outcome. Wolfe [13], for example, developed an AHP model to adjust the forecasts of earnings per share of firms. The factors considered as critical to the forecast are economy, historical (accounting data), product line, and fill-in factor. The fill-in factor is a special element so that different firms (or forecasters) can add their own criterion. He tested his model using doctoral students in finance and Certified Public Accountants (CPAs) as the forecasters. The students were near degree completion, and the CPAs had big-firm public accounting experience. The overall outcome was inconclusive, but he found that CPAs were able to come to the correct forecasts. The results emphasized that experts make the difference.

\section{Conclusion}

I have examined three ideas in the paper. The first idea is that expectations about the outcome of a decision in the AHP can be satisfied by different ways of combining the final answer. One should not be hasty to judge the outcome without understanding the purpose for which it was derived. The second idea is that mechanically grouping elements into clusters does not automatically create new criteria with a meaning that fits with other parts of the hierarchy. Otherwise by simply changing the number of groups to which different alternatives belong, one can influence their relative importance. The third idea is that measurements on an absolute scale must be unified under a single overall criterion, normalized, then combined with other scales for the outcome to be meaningful. Alternatively, pairwise comparisons based on importance, preference, or likelihood can be applied to the data to derive the necessary relative scales.

\section{References}

[1] Bernhard, R. H. and Canada, J. R., "Some problems in using benefit/cost ratios with the Analytic Hierarchy Process: Technical notes", The Engineering Economist 36(1) Fall 1990.

[2] Forman, E. H., "AHP is intended for more than expected value calculations", Decision Sciences 21 (1990) 670-673.

[3] Hauser, D. and Peniwati, K, "Misuse of the axiom of independence in the Analytic Hierarchy Process", Proc. 3rd Int. Symp. on the Analytic Hierarchy Process, School of Business and Public Management, The George Washington University, Washington, DC, 1994. 
[4] Saaty, T. L., The Analytic Hierarchy Process, New York, NY, McGraw-Hill Book Company, Inc., 1980.

[5] Saaty, T. L., Decision Making for Leaders, RWS Publication, Pittsburgh, 1990.

[6] Saaty, T. L., Multicriteria Decision Making: The Analytic Hierarchy Process, RWS Publication, Pittsburgh, 1990.

[7] Saaty, T. L. Fundamentals of Decision Making and Priority Theory, RWS Publication, Pittsburgh, 1994.

[8] Saaty, T. L. and Kearns, K., Analytical Planning, RWS Publication, Pittsburgh, PA, 1985.

[9] Schenkerman, S., "Use and abuse of weights in multiple objective decision support models", Decision Sciences 22 (1991) 369-378.

[10] Schoner, B. and Wedley, W. C., "Ambiguous criteria weights in AHP: consequences and solutions", Decision Sciences 20 (1989) 462-475.

[11] The Economist, March 12th, 1994, 93-94.

[12] Wedley, W. C., Schoner, B., and Choo, E. U., "Clustering, dependence and ratio scales in AHP: Rank reversals and incorrect priorities with a single criterion", Journal of Multicriteria Decision Analysis 2 (1993) 145-158.

[13] Wolfe, C., "How to adjust forecasts with the Analytic Hierarchy Process", The Journal of Business Forecasting Spring 1988, 13-17. 